\title{
Auditory presentation leads to better analogical retrieval than written presentation
}

\author{
Arthur B. Markman \\ University of Texas, Austin, Texas \\ ERIC TAYLOR \\ University of Illinois, Urbana, Illinois \\ AND \\ DEDRE GENTNer \\ Northwestern University, Evanston, Illinois
}

\begin{abstract}
Research on analogical retrieval suggests that cues with object similarity to a prior episode in memory lead to better retrieval than do cues with relational similarity. We suggest that previous work may have underestimated the effectiveness of relational cues, because this work has presented cues and targets in written format. There is some evidence that spoken presentations lead to better memory than do written presentations. We tested this hypothesis using a continuous reminding paradigm in which people read and recalled proverbs that were presented either in spoken or written format. The spoken format led to better retrieval from relational cues, particularly at longer lags between cue and memory item.
\end{abstract}

Analogical retrieval is crucial for reasoning by analogy. Although there are cases in which people are given two domains and are asked to reason about one (the target) by virtue of its similarity to a second (the base), useful analogies are often retrieved spontaneously during reasoning and problem solving. For this reason, researchers have been interested in the conditions under which people are able to retrieve good base domains given a target cue (e.g., Dunbar, 1997; Gentner, Rattermann, \& Forbus, 1993; Gick \& Holyoak, 1980; Hammond, Seifert, \& Gray, 1991; Wharton, Holyoak, \& Lange, 1996).

Research on analogical reasoning suggests that a useful analogy between a base and a target occurs when the domains share systematic relational similarities. Relations are representational elements of a domain that link the entities, descriptive attributes, and other relations. Relations that connect other relations are called higher-order relations, and systems of relations linked by such relations are thought to be particularly important for reasoning (Clement \& Gentner, 1991). When the relational structure of two domains matches, they are analogous. Most analogies involve cases in which there are relational matches between the domains, but the entities in the domains themselves are not similar (i.e., they have little attribute similarity; Gentner, 1983, 1989).

Relational similarity can be illustrated using proverbs. The proverb "The swiftest steed can stumble" is relationally similar to the proverb "The greatest master is wrong from time to time," because both have the (relational) meaning that everyone, even the powerful, can make mistakes. In contrast, the proverb "A rough steed needs a rough bridle" shares only attribute similarity with the first proverb, because both are about steeds. The meanings of the proverbs are not similar.

People are quite good at detecting analogical similarities when two situations (or proverbs) are juxtaposed. Furthermore, people find analogous situations useful for reasoning. Nonetheless, a striking finding from research on analogical retrieval is that people often fail to retrieve items that are relationally similar to a cue when they have them in memory, even if those prospective analogies would be useful for solving a problem (Gentner et al., 1993; Ross, 1987, 1989). For example, in a classic study, Gick and Holyoak (1980) observed that only about $30 \%$ of subjects could solve an insight problem even when given a prior analogous story. Of importance, almost all subjects were able to retrieve and use the analogue when given a hint to use it. This result suggests that the failure to use the analogous story from memory was a failure to retrieve that story rather than an inability to use the story once retrieved.

The existing literature on analogical retrieval can be summarized by two generalizations. First, cues with primarily object similarity to an item in memory are much more effective retrieval cues than are cues with primarily relational similarity. Obviously, cues with both object and relational similarity are also effective retrieval

A. B. Markman, markman@psy.utexas.edu 
cues, though not typically more effective than those with only object similarity (Catrambone, 2002; Gentner et al., 1993). Second, relational retrieval is improved by conditions that favor relational encoding of the memory item and the probe, such as the use of experimental manipulations that invite comparing two analogous memory items (Catrambone \& Holyoak, 1989; Gentner, Loewenstein, \& Thompson, 2003; Gick \& Holyoak, 1983).

Despite the result that relational similarity is less a effective retrieval cue than object similarity, there is a persistent belief that analogies are used by good problem solvers (Hammond et al., 1991; Polya, 1945; Schank, 1990). Novick (1988) showed better relational retrieval for mathematics problems in relatively expert mathematics students than in novice mathematicians. Dunbar (1997) found that scientists are able to use analogies, and some of them come from relationally distant domains. Furthermore, Blanchette and Dunbar (2001) have demonstrated that analogies are used in a variety of natural settings, meaning that these analogies had to be retrieved in some fashion.

How can we reconcile the inability of people to retrieve analogies in laboratory studies with the sense that people can and do retrieve analogies? The possibility we explore in this article is that the literature has underestimated people's ability to retrieve analogies because of the way that studies of analogical retrieval are normally conducted. In the typical experiment, subjects read a base domain (which may consist of a proverb or a story). After a retention interval, they read a target cue and must recall a prior instance either by writing down the information they recall or speaking aloud their remindings.

The studies we are aware of all involve written stories and cues. In this article, we explore the hypothesis that written presentations underestimate people's analogical retrieval ability. There is little research on modality differences for memory or comprehension of complex text, but we found some related work that is consistent with this hypothesis. For example, research in list learning suggests that spoken presentations of lists lead to better memory for words than do written presentations (e.g., Conway \& Gathercole, 1987). However, we know of no research examining modality effects on relational retrieval directly.

The hypothesis that spoken presentation of information might better enable relational recall than written presentation stems from evidence suggesting that people are better able to rapidly form complex interpretations of spoken text than of written text. For example, Jakimik and Glenberg (1990) found that people are better able to resolve anaphors (which require them to resolve the temporal structure of the text) for spoken text than for written text. Carroll and Korukina (1999) found better memory for elements of complex spoken text than for written text. These results suggest that comprehension and memory for relational items may be better for spoken presentations than for written presentations.

Of course, the possibility that people can more readily derive complex representations of spoken text than of written text is somewhat counterintuitive. One might expect that the written modality would better support careful, deliberative processing and would thus foster deeper encodings than would spoken text.
To explore the possibility that previous studies have underestimated people's analogical retrieval abilities, we replicated a previous study involving memory for proverbs (Schumacher \& Gentner, 1987) and manipulated whether the proverbs were presented in written or spoken format. The Schumacher and Gentner study (described in Gentner, Bowdle, Wolff, \& Boronat, 2001) used a continuous reminding design in which people were exposed to a series of proverbs. On some trials, people were asked to define the proverb they heard. (This was done only on filler items, not on experimental items.) These instructions were given to focus people on the conceptual and relational information in the proverbs. On the key experimental trials, they were asked to recall any proverb they had heard during the study that was similar to the cue.

The key items were 24 pairs of proverbs. One third of these pairs involved relational similarities (as in the steed/ master pair described above). Another third contained an object similarity (as in the pair of proverbs involving steeds). In the final third, the two proverbs were identical. In these pairs, the base proverb always occurred earlier in the list. The cue could appear one, six or eleven items later in the list. Schumacher and Gentner (1987) found that people were far better at recalling object-similar proverbs than relationally similar proverbs (and, as expected, they were nearly perfect on the identical pairs).

In our studies, we were interested in the degree to which people would recall proverbs given cues with object or relational similarities. Schumacher and Gentner's (1987) findings, consistent with other analogical retrieval results, suggest that people will recall more proverbs given an object similarity cue than given a relational similarity cue. However, if spoken language better enables relational recall than written language, then we should observe more analogical retrieval in the spoken condition than in the written condition.

\section{METHOD}

The subjects were 89 members of the University of Texas community who received course credit or were paid $\$ 8$ for their participation. Overall, 45 subjects were run in the written condition and 44 in the spoken condition. Subjects were run individually by an experimenter.

The proverbs used in this study were adapted from previous research by Schumacher and Gentner (1987). We constructed three lists of proverbs, and each subject was exposed to one of those lists. The lists consisted of 12 proverb pairs that shared object similarity and 12 proverb pairs that shared relational similarity (for a total of 48 proverbs) along with 28 filler proverbs that did not share either object or relational similarity with the rest of the pairs in the set. Each list had a base proverb paired with a different proverb that shared either relational or object similarity with it, in order to ensure that the results were not due to a specific pairing between particular proverbs. The lists were constructed so that one pair at each distance appeared in each third of the list.

Each list began with three filler proverbs. The base proverbs always preceded the cue proverbs. Cues were presented 1, 2, 4, or 8 proverbs later in the list (that is, at a distance of 1,2,4, or 8). Prior to the presentation of a proverb, subjects were told either that they were going to define that proverb or that they were to use it to recall previously presented proverbs. The subjects defined 13 of the filler proverbs (including the first two in the set). They were given instructions to recall previous proverbs for all other items. 
Subjects in the written condition were shown each proverb on an index card. Reading was self-paced. Subjects in the spoken condition heard a recorded reading of each proverb, spoken by a female who did not know the purpose of the study. Those in the spoken condition were allowed to hear the proverb as many times as they wanted. The subjects in both conditions spoke aloud their definition or what they recalled (depending on the instructions for a trial).

Sessions were audiotaped. The number of base proverbs correctly recalled was recorded for all subjects by one of the authors. Recall data from 34 subjects ( 18 in the spoken condition and 16 in the written condition) was scored by a second rater. The two raters agreed on $98.4 \%$ of their coding. For the definition trials, there were a few cases in which subjects failed to provide a definition. These trials were not included in the analyses. However, all subjects provided a definition for at least 11 of the 13 proverbs. One of the authors scored the definitions of 64 subjects ( 29 from the written condition and 35 from the spoken condition) $)^{1}$ for the degree to which they captured surface and relational aspects of the proverb. The definitions of 51 subjects ( 26 written and 25 spoken) were also coded by a second rater, who was naive to our experimental hypothesis. Use of surface information was rated on a scale from 1 to 4 , where 1 meant that the definition used no words from the proverb and 4 meant that most of the words from the proverb were repeated in the definition. The relationality of the definition was also scored on a $1-4$ scale, where 1 meant that the subject provided only a shallow definition and 4 that the subject gave a good definition of the relational meaning. Cohen's weighted $\kappa$ (a measure of reliability for ordinal data; Cohen, 1968) indicated moderate agreement for both object $(\kappa=$ $.62)$ and relational $(\kappa=.72)$ information. The pattern of data for all analyses involving these definitions is the same regardless of which coder's data is used. The analyses we present were performed on the 64 subjects mentioned above.

To summarize, this study had a 2 (modality: written vs. spoken) $\times$ 2 (match type: relational vs. object) $\times 4$ (distance: $1,2,4$, or 8 ) design. Modality was run between subjects and match type and distance were run within subjects. In preliminary analyses, the proverb list was included as a factor. Because this factor did not influence the results, it will not be discussed further.

\section{RESULTS AND DISCUSSION}

The primary data of interest are the mean proportion of proverbs of each type correctly recalled at each distance for the two modalities. Subjects rarely recalled proverbs incorrectly, so we focus only on correct recalls. The recall data are shown in Figure 1. A 2 (modality) $\times 2$ (match type) $\times$ 4 (distance) mixed-model ANOVA revealed the expected main effect of match type $\left[F(1,87)=13.15, p<.05, \eta^{2}=\right.$ $.13]$, reflecting that a higher proportion of proverbs were recalled for cues that were an object match $(M=.46)$ than for cues that were a relational match $(M=.31)$. There was also a reliable main effect of distance $[F(3,261)=12.26$, $\left.p<.05, \eta^{2}=.12\right]$, reflecting that fewer proverbs were recalled as the distance between the cue and the target increased. ${ }^{2}$ In addition, there was a significant interaction between match type and distance, indicating that the effect of distance was more pronounced for relational matches than for object matches $\left[F(3,261)=3.32, p<.05, \eta^{2}=\right.$ .04]. So far, these findings mirror those of Schumacher and Gentner (1987).

Of central importance to our predictions, though, is a significant three-way interaction between modality, match type, and distance $\left[F(3,261)=2.86, p<.05, \eta^{2}=.03\right]$ that qualifies both the main effect of distance and the match type $\times$ distance interaction. As shown in Figure 1, this interaction reflects a difference in the recall of relational versus object matches across the two modalities. The proportion of object matches recalled for written and spoken proverbs was roughly the same at all distances. Planned comparisons were done using $t$ tests comparing the mean numbers of object matches recalled at the various distances revealed no reliable differences as a function of the modality of presentation (all $p \mathrm{~s}>.2$ ).

The pattern for relational recall is quite different. As predicted, a higher proportion of proverbs was recalled for cues with a relational match for spoken proverbs than for written proverbs at all distances. This difference was significant for distances of $4[M=.21$ written and $M=$ .33 spoken; $t(87)=2.29]$ and $8[M=.12$ written and $M=.29$ spoken; $t(87)=2.85$ ] (both $p$ s $<.05$, Bonferroni corrected).

One possible explanation for these results is that people encoded the proverbs more effectively in the spoken condition than in the written condition. To explore this possibility, we analyzed the quality of the definitions people gave for those proverbs they were asked to define. As discussed above, we coded the use of surface and relational information in the definitions. The mean ratings of surface and relational information for proverbs in the spoken and written conditions are shown in Table 1. Subjects in the written condition used significantly more surface information in their proverbs $(M=1.99)$ than did subjects in the spoken condition $(M=1.68)[t(62)=3.32, p<$ $.05]$. There was no significant difference in the amount of relational information between the spoken $(M=2.33)$ and written $(M=2.22)$ conditions $[t(62)=1.27, p>$ .10]. However, the interaction between surface/relational information and condition was also significant $[t(62)=$ $25.63, p<.05]$, suggesting that spoken condition subjects may have allocated attention rather more to relational and less to surface information, as compared with the written condition subjects. There was no significant correlation between the mean use of surface and relational information within subjects $[r(62)=-.04, p>.10]$.

Of course, the proverbs that subjects defined were not the same as the ones that they recalled. Nonetheless, these ratings may reflect the quality of people's comprehension for all proverbs they saw. Thus, for each subject, we correlated the mean use of surface and relational information in the definitions with the mean recall of relational and object match proverbs. Neither measure of definition quality correlated significantly with recall of object match proverbs $[r(62)=.02$ for use of surface information, $r(62)=$ -.07 for use of relational information; both $p \mathrm{~s}>.10]$. The correlation between use of surface information and recall of relational matches was also nonsignificant $[r(62)=$ $.02, p>.10]$. However, there was a significant correlation between the use of relational information in definitions and recall of relational matches $[r(62)=.61, p<$ $.05]$ : That is, individuals who tended to extract relational definitions of proverbs showed superior ability to recall (other) proverbs on the basis of relational information. This finding suggests that attention to relations during encoding leads to increased ability to recall on the basis of relational matches. 

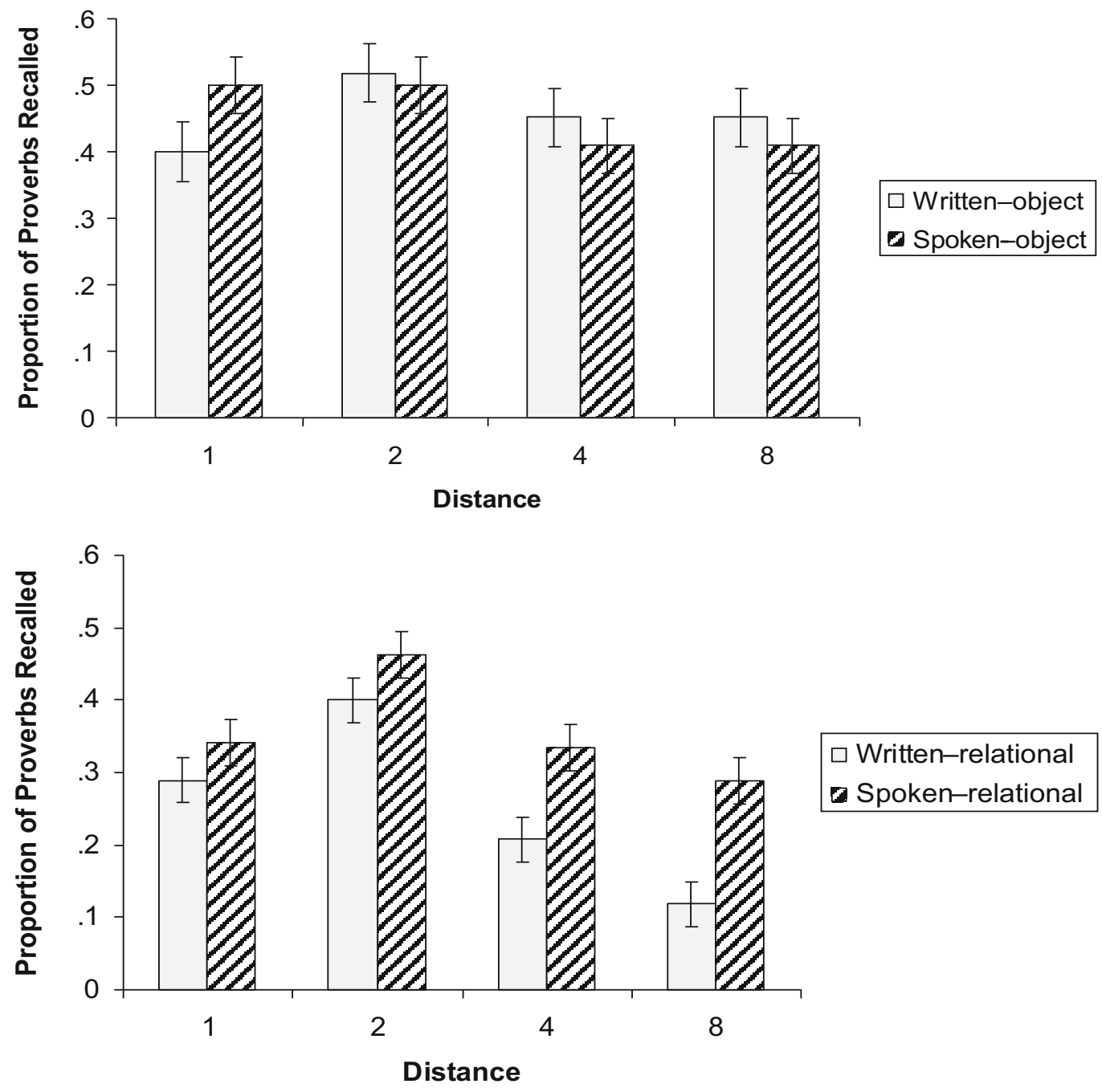

Figure 1. Mean proportions of proverbs recalled in the experiment as a function of the modality of presentation, type of match, and distance between the cue and target. The top panel shows recall of object matches, and the bottom panel shows recall of relational matches. Error bars are one standard deviation from the mean.

How might this be related to the observed modality difference? We determined the ratio of the relational quality of the definitions to the surface quality for each subject and correlated that with their recall data. This ratio correlated significantly with recall of relational matches $[r(62)=.27$, $p<.05]$, but not with recall of object matches $[r(62)=$ $.00, p>.10]$. Hence, subjects who provided more relational information, relative to surface information, tended to recall more relational match proverbs.

To summarize the analysis of the definitions, the quality of the relational information in the definitions was similar for both spoken and written presentation, but there was more surface information in the definitions for written proverbs than for spoken proverbs. The amount of surface information in definitions did not correlate with retrieval of proverbs, but the amount of relational information in the definitions was positively correlated with subjects' ability to retrieve based on relational similarities.
The correlation between the quality of relational definitions and amount of relational retrieval is consistent with other research demonstrating that relational encoding has a positive influence on relational retrieval. For example, a number of studies have shown that comparing two analogous texts during study - which promotes extraction of the common relations (Gentner et al., 2003; Gick \& Holyoak, 1983) — can lead to better relational transfer than does

Table 1

Mean Surface and Relationality Ratings for the Proverbs in the Spoken and Written Conditions

\begin{tabular}{lccccc}
\hline & \multicolumn{2}{c}{$\begin{array}{c}\text { Spoken } \\
\text { Proverbs }\end{array}$} & & \multicolumn{2}{c}{$\begin{array}{c}\text { Written } \\
\text { Proverbs }\end{array}$} \\
\cline { 2 - 3 } \cline { 5 - 6 } \multicolumn{1}{c}{ Rating Type } & $M$ & $S D$ & & $M$ & $S D$ \\
\hline Surface information & 1.68 & 0.35 & & 1.99 & 0.37 \\
Relational information & 2.33 & 0.35 & & 2.22 & 0.37 \\
\hline
\end{tabular}


reading the two texts separately (Catrambone \& Holyoak, 1989; Gentner et al. 2003; Gick \& Holyoak, 1983; Loewenstein, Thompson, \& Gentner, 1999). Research by Clement, Mawby, and Giles (1994) also indicates that the nature of the relational encoding matters; they found better retrieval to a relational probe when the relations in the memory passage were phrased in a domain-general way than when they were phrased in a context-specific way. They suggested that the domain-general phrasing made the relational match between target and probe more apparent. This finding is also consistent with the suggestion that experts encode materials using a uniform set of relations (Forbus, Gentner, \& Law, 1995), leading to better relational retrieval in experts than in novices (Novick, 1988). There is also evidence that asking people to produce analogies rather than to recall passively presented analogies leads to significant analogical retrieval (Blanchette \& Dunbar, 2000).

Previous research has concluded that cues with object similarity to an item in memory are significantly more effective than cues with relational similarity (e.g., Catrambone, 2002; Gentner et al., 1993; Holyoak \& Koh, 1987). Furthermore, these studies suggest that relational remindings are rare. Our data are consistent with these general conclusions: In both spoken and written modalities, object similarity was a more effective retrieval cue than relational similarity. However, the results of this study further indicate that prior work may have underestimated people's ability to retrieve on the basis of relational similarity. Building on previous research that has focused on written materials, our results suggest that spoken materials lead to more relational remindings, particularly at higher lags between cue and target.

This research adds to a body of knowledge concerning factors that can improve levels of analogical retrieval. For example, Wharton and colleagues found that people were more likely to retrieve an item with a relational match to a cue when there was also a competing object match to the cue in memory (Wharton et al., 1994). Catrambone (2002) demonstrated that relational cues are most effective when they contain a higher-order relational similarity that binds together the individual relations of a story rather than containing a set of disconnected relational elements. Likewise, Gentner et al. (1993) found that relational cues that shared higher-order relations with the prior exemplar were more effective cues than those that shared only firstorder relations. Finally, as discussed above, comparison of two initial analogues (Catrambone \& Holyoak, 1989; Gentner et al., 2003; Gick \& Holyoak, 1983) has been shown to lead to greater relational retrieval.

Our findings add a new factor: They suggest that the modality of processing can influence ease of relational reminding. This study was not designed to tease apart the locus of the modality effect, but previous research has suggested that modality differences can affect both encoding and retrieval processes. On the encoding side, there is evidence that spoken presentations may lead to better comprehension and memory than written (Carroll \& Korukina, 1999; Conway \& Gathercole, 1987; Jakimik \& Glenberg, 1990). The definition data suggest a speculative possibility: that spoken presentation promotes a relatively greater focus on relational information in comparison to surface information than does written presentation. This difference in encoding focus would lead to an increased likelihood of relational remindings among the spoken condition subjects.

Why might such a difference in encoding focus come about? One possibility is that people are more practiced at rapid extraction of the main point in the spoken discourse than in written discourse, for which backtracking is generally possible. This would be consistent with evidence that subjects in spoken dialogues are able (in most circumstances) to quickly arrive at a shared understanding of the topic of the conversation - that is, to establish a level of common ground (Clark, 1996; Horton \& Gerrig, 2005). A related possibility is that discourse-level routines (such as extracting the point of a proverb) might be more readily invoked during spoken input than during written input.

On the retrieval side, it is possible that reading causes a higher memory load than does spoken language comprehension, because written text must be converted to a phonological code prior to comprehension (see, e.g., Rayner $\&$ Clifton, 2002). Previous research on analogical reasoning suggests that analogical reasoning is a computationally intensive process that makes strong demands on working memory (Falkenhainer, Forbus, \& Gentner, 1989; Hummel \& Holyoak, 1997). Holyoak and his colleagues have demonstrated that the ability to make relational mappings is impaired by a memory load (Tohill \& Holyoak, 2000; Waltz, Lau, Grewal, \& Holyoak, 2000). On this view, the difference in working memory demands of written and spoken language comprehension would favor analogical retrieval for spoken presentations of retrieval cues.

The present study does not allow us to decide between these possibilities. However, the phenomenon of greater relational retrieval in oral communication is from many points of view counterintuitive and intriguing. Future research must explore the degree to which encoding focus and/or working memory differences between processing of written and spoken text are at the root of the observed differences in relational retrieval.

\section{AUTHOR NOTE}

This work was supported by NIH Grant R21-DA015211 to the first author. The authors thank Leora Orent for recording the proverbs and for serving as a second rater on the data. Correspondence concerning this article should be addressed to A. B. Markman, Department of Psychology, University of Texas, One University Station, A8000, Austin, TX 78712 (e-mail:markman@psy.utexas.edu).

\section{REFERENCES}

Blanchette, I., \& Dunbar, K. (2000). How analogies are generated: The roles of structural and superficial similarity. Memory \& Cognition, 28, 108-124.

Blanchette, I., \& Dunbar, K. (2001). Analogy use in naturalistic settings: The influence of audience, emotion, and goals. Memory \& Cognition, 29, 730-735.

Carroll, M., \& Korukina, S. (1999). The effect of text coherence and modality on metamemory judgements. Memory, 7, 309-322.

Catrambone, R. (2002). The effects of surface and structural feature matches on the access of story analogs. Journal of Experimental Psychology: Learning, Memory, \& Cognition, 28, 318-334.

Catrambone, R., \& Holyoak, K. J. (1989). Overcoming contextual 
limitations on problem-solving transfer. Journal of Experimental Psychology: Learning, Memory, \& Cognition, 15, 1147-1156.

Clark, H. H. (1996). Using language. New York: Cambridge University Press.

Clement, C. A., \& Gentner, D. (1991). Systematicity as a selection constraint in analogical mapping. Cognitive Science, 15, 89-132.

Clement, C. A., Mawby, R., \& Giles, D. E. (1994). The effects of manifest relational similarity on analog retrieval. Journal of Memory \& Language, 33, 396-420.

Cohen, J. (1968). Weighted kappa: Nominal scale agreement with provision for scaled disagreement or partial credit. Psychological Bulletin, 70, 213-220.

Conway, M. A., \& Gathercole, S. E. (1987). Modality and long-term memory. Journal of Memory \& Language, 26, 341-361.

Dunbar, K. (1997). How scientists think: On-line creativity and conceptual change in science. In T. B. Ward, S. M. Smith, \& J. Vaid (Eds.), Creative thought: An investigation of conceptual structures and processes (pp. 461-493). Washington, DC: American Psychological Association.

Falkenhainer, B., Forbus, K. D., \& Gentner, D. (1989). The structuremapping engine: Algorithm and examples. Artificial Intelligence, 41, $1-63$.

Forbus, K. D., Gentner, D., \& LAW, K. (1995). MAC/FAC: A model of similarity-based retrieval. Cognitive Science, 19, 141-205.

GentNer, D. (1983). Structure-mapping: A theoretical framework for analogy. Cognitive Science, 7, 155-170.

GentNER, D. (1989). The mechanisms of analogical learning. In S. Vosniadou \& A. Ortony (Eds.), Similarity and analogical reasoning (pp. 199-241). New York: Cambridge University Press.

Gentner, D., Bowdle, B., WolfF, P., \& Boronat, C. (2001). Metaphor is like analogy. In D. Gentner, K. J. Holyoak, \& B. N. Kokinov (Eds.), The analogical mind (pp. 199-253). Cambridge, MA: MIT Press.

Gentner, D., Loewenstein, J., \& Thompson, L. (2003). Learning and transfer: A general role for analogical encoding. Journal of Educational Psychology, 95, 393-408.

Gentner, D., Rattermann, M. J., \& Forbus, K. D. (1993). The roles of similarity in transfer: Separating retrievability from inferential soundness. Cognitive Psychology, 25, 524-575.

Gick, M. L., \& HolyonK, K. J. (1980). Analogical problem solving. Cognitive Psychology, 12, 306-355.

Gick, M. L., \& Holyoak, K. J. (1983). Schema induction and analogical transfer. Cognitive Psychology, 15, 1-38.

Hammond, K. J., Seifert, C. M., \& Gray, K. C. (1991). Functionality in analogical transfer: A hard match is good to find. Journal of the Learning Sciences, 1, 111-152.

HolyOAK, K. J., \& KoH, K. (1987). Surface and structural similarity in analogical transfer. Memory \& Cognition, 15, 332-340.

Horton, W. S., \& Gerrig, R. J. (2005). The impact of memory demands upon audience design during language production. Cognition, 96, 127-142.

Hummel, J. E., \& Holyoak, K. J. (1997). Distributed representations of structure: A theory of analogical access and mapping. Psychological Review, 104, 427-466.
JAKIMIK, J., \& GLENBERG, A. (1990). Verbal learning meets psycholinguistics: Modality effects in the comprehension of anaphora. Journal of Memory \& Language, 29, 582-590.

Loewenstein, J., Thompson, L., \& Gentner, D. (1999). Analogical encoding facilitates knowledge transfer in negotiation. Psychonomic Bulletin \& Review, 6, 586-597.

Novick, L. R. (1988). Analogical transfer, problem similarity, and expertise. Journal of Experimental Psychology: Learning, Memory, \& Cognition, 14, 510-520.

Polya, G. (1945). How to solve it. Princeton, NJ: Princeton University Press.

Rayner, K., \& Clifton, C. (2002). Language processing. In H. Pashler \& D. L. Medin (Eds.), Stevens' Handbook of psychology (Vol. 2, pp. 261-316). New York: Wiley.

Ross, B. H. (1987). This is like that: The use of earlier problems and the separation of similarity effects. Journal of Experimental Psychology: Learning, Memory, \& Cognition, 13, 629-639.

Ross, B. H. (1989). Distinguishing types of superficial similarities: Different effects on the access and use of earlier examples. Journal of Experimental Psychology: Learning, Memory, \& Cognition, 15, 456-468.

SCHANK, R. C. (1990). Tell me a story: Narrative and intelligence. Evanston, IL: Northwestern University Press.

Schumacher, R., \& Gentner, D. (1987, May). Similarity-based remindings: The effects of similarity and interitem difference. Paper presented at the annual conference of the Midwestern Psychological Association, Chicago, IL.

TohILl, J. M., \& HolYOAK, K. J. (2000). The impact of anxiety on analogical reasoning. Thinking \& Reasoning, 6, 27-40.

Waltz, J. A., Lau, A., Grewal, S. K., \& Holyoak, K. J. (2000). The role of working memory in analogical mapping. Memory \& Cognition, 28, 1205-1212.

Wharton, C. M., Holyoak, K. J., Downing, P. E., Lange, T. E., Wickens, T. D., \& Melz, E. R. (1994). Below the surface: Analogical similarity and retrieval competition in reminding. Cognitive Psychology, 26, 64-101.

Wharton, C. M., Holyoak, K. J., \& Lange, T. E. (1996). Remote analogical reminding. Memory \& Cognition, 24, 629-643.

\section{NOTES}

1. Because of a clerical error, 25 audio tapes were lost before the analysis of the definitions was carried out, leaving 64 that were analyzed.

2. Surprisingly, relational recall was better for proverbs at Distance 2 than at Distance 1 for both the spoken and written conditions. This finding was unexpected, and we found no obvious precedent for it in the literature. This pattern should be explored in future research. It does not compromise the conclusions drawn here, however, because relational recall was still better in the spoken condition than in the written condition.

(Manuscript received May 30, 2006; revision accepted for publication March 9, 2007.) 